\title{
Fortalecer la investigación como estrategia pedagógica a través de la lectura y escritura mediante figuras literarias ${ }^{1}$
}

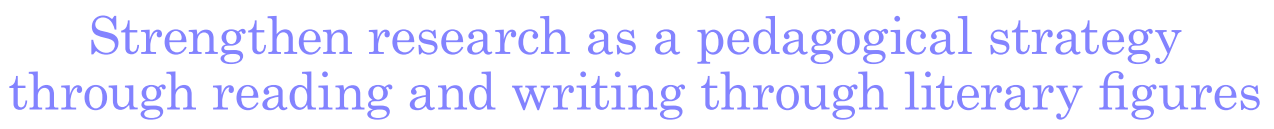

DOI: http://dx.doi.org/10.17981/cultedusoc.9.3.2018.110

Artículo de investigación. Fecha de recepción: 15/06/2018. Fecha de aceptación: 27/11/2018

\author{
Carmen Barrios-García ${ }^{2}$ \\ Genny Berdugo-Ospino; María Sierra-Navarro; Nilson Cáceres-Hoyos; \\ Jorge Chamorro-Marimon; Juan Meriño-Canaval y Ana Sánchez Gómez ${ }^{3}$ \\ Escuela Urbana Mixta Policarpa Salavarrieta (Colombia) \\ jcperlaza@yahoo.es
}

Para citar este artículo:

Barrios-García, C., Berdugo-Ospino, G., Sierra-Navarro, M., Cáceres-Hoyos, N., Chamorro-Marimon, J., Meriño-Canaval, J. y Sánchez, A. (2018). Fortalecer la investigación como estrategia pedagógica a través de la lectura y escritura mediante figuras literarias. Cultura. Educación y Sociedad 9(3), 929-936. DOI: http://dx.doi.org/10.17981/cultedusoc.9.3.2018.110

\section{Resumen}

En los últimos tiempos ha aumentado el grado de dificultad para enseñar en las instituciones de educación básica, pues los niños y niñas han perdido el interés por adquirir conocimientos y desvían su atención a actividades distintas; por esto ha surgido la necesidad de implementar modelos pedagógicos que puedan ser interactivos y didácticos. El estudio tuvo como objetivo fortalecer la investigación como estrategia pedagógica (IEP) para a través de la lectura y escritura mediante figuras literarias. Estuvo guiado bajo los lineamientos de la investigación cualitativa, utilizando técnicas propias de este tipo de investigación necesaria para la recolección de información como la observación participante y herramientas como el diario de campo, la unidad de análisis estuvo conformada por ciento treinta (130) estudiantes con edades que oscilaban entre los siete (7) y once (11) años de la Institución Educativa Departamental Gabriel Escobar Ballestas. Los resultados permitieron observar que la IEP logró despertar el interés de los estudiantes por la investigación y además capacitarlos para crear sus propios cuentos donde expresen lo que ellos quisieran, vivencias, o imaginario con la finalidad de fortalecer la investigación a través de las figuras literarias.

Palabras clave: investigación como estrategia pedagógica, lectura, escritura, figuras literarias.

\section{Abstract}

In recent times the degree of difficulty in teaching in basic education institutions has increased, as children have lost interest in acquiring knowledge and divert their attention to different activities; This is why the need to implement pedagogical models that can be interactive and didactic has arisen. The objective of the study was to strengthen research as a pedagogical strategy (IEP) for reading and writing through literary figures. It was guided under the guidelines of qualitative research, using techniques of this type of research necessary for the collection of information such as participant observation and tools such as the field diary, the analysis unit consisted of one hundred and thirty (130) students with ages that oscillated between the seven (7) and eleven (11) years of the Departmental Educational Institution Gabriel Escobar Ballestas. The results allowed observing that the IEP managed to awaken students' interest in research and also train them to create their own stories where they express what they want, experiences, or imaginary in order to strengthen research through literary figures.

Keywords: Didactic strategy, learning, promotion of research.

1 Este artículo ha sido derivado del Programa de Fortalecimiento de la Cultura Ciudadana y Democrática CT+I a través de la IEP apoyada en TIC en el Departamento de Magdalena: CICLON

2 Líder del grupo de investigación "Gips Policarpa Salavarrieta".

3 Docentes de la Escuela Urbana Mixta Policarpa Salavarrieta.

- The author; licensee Universidad de la Costa - CUC.

Cultura, Educación y Sociedad vol. 9 no. 3, pp. 929-936. Diciembre, 2018

Barranquilla. ISSN 2389-7724 Online 


\section{Introducción}

En la actualidad, existen propuestas metodológicas de enfoques basados en investigación, entendidos como la forma de materializar lo que se hace y como se implementa donde surgen infinidad de propuestas metodológicas que se basan en un enfoque o toman varios para hacer de ellos una propuesta metodológica; es el caso de las pedagogías basadas o fundadas en investigación, las cuales se desarrollan en diferentes contextos y asumen variados caminos en coherencia con los paradigmas y corrientes en los cuales se inscriben. Dentro de esta teoría basada en investigación se encuentra la Enseñanza por descubrimiento y el Programa Ondas, con su propuesta de la Investigación como Estrategia Pedagógica (IEP), como una perspectiva que se desarrolla en ese enfoque y toma elementos de la pedagogía critico-liberadora y del enfoque sociocultural (Mejía y Manjarrés, 2010).

Así, considerar la investigación como estrategia pedagógica implica recrear un espacio de inclusión, donde se respete y se viva la diversidad (Carvajal, 2013) haciendo evidentes los canales de la creatividad del docente, para convertir las preguntas de los grupos de aprendizaje colaborativo conformados por niños, niñas y adolescentes en una investigación, llevándolos a tomar un rol activo en el proceso de aprendizaje mediante la construcción de su propia conocimiento, potenciando así, las habilidades y destrezas de los estudiantes, convirtiendo la educación en un vehículo de capacitación, de oportunidades y de recreación (Camargo, 2015). Se desprende de lo antes planteado el rol activo del docente en el proceso de mediación; así, Pinto y Misas (2014) destacan que los profesores contribuyen a desarrollar capacidades en los estudiantes para pensar y actuar con autonomía y participar en la toma de decisiones.
Además, la investigación como estrategia pedagógica, busca lograr que asesores, maestros y maestras, niños, niñas y jóvenes, establezcan un dialogo de saberes mediado por una formación en investigación, que integrada al aula de clase permita cultivar el espíritu científico en los estudiantes desde edades tempranas, creando una cultura de investigación, que a su vez posibilite una educación basada en ciencia tecnología e innovación, convirtiéndose en un aporte para el desarrollo del país (Santacoloma, 2014).Al respecto Pino y Urrego (2013), sostienen que cualquier proceso de desarrollo considera la integración individuo-sociedad y las relaciones que se dan entre los actores sociales, todo ello en el ámbito del desarrollo de competencias para vivir en ciudadanía. En concordancia con lo planteado Moreno (2015) considera el contexto social como un ámbito dinámico donde las relaciones entre los individuos encuentran razón de ser y es precisamente la dinámica entre los ciudadanos que permite la construcción colectiva del referido contexto.

En este orden de ideas se enfatiza que la propia dinámica social lleva a que los individuos, especialmente los más jóvenes dirijan su atención a situaciones diferentes a las educativas, esto es debido a que perciben la educación como algo monótono, por esto es necesario crear nuevas ideas que permitan hacer la clase mucho más didáctica, atrayendo la atención de los estudiantes y permitiendo la transmisión del conocimiento en un aula de clase. Por esto se recurre a la utilización de la leyenda del hombre caimán como estrategia pedagógica que promueva la enseñanza, investigación y cultura en el departamento de Magdalena (Giroux, 2003).

La Pedagogía es una ciencia muy compleja que debe ser vista con cuidado, debido a que la pedagogía estudia la educación y la apropiación de los valores de 
conocimiento en el ser humano. La palabra Pedagogía hace referencia a orientación, visión, evocada puntualmente a la formación de métodos que peritan desarrollar una educación de calidad. La pedagogía en una institución es vista como el proceso analítico de las técnicas y métodos de enseñanza que existen dentro y fuera del plantel educativo, para reforzar o elaborar mejores estrategias que el ayudaran a los estudiantes a la adquisición de mayor conocimiento (Uribe, 2002; Benito, 2002; Freire, 2005).

La Pedagogía es hoy en día un término mundialmente conocido, teniendo en cuenta las nuevas generaciones y las nuevas tecnologías que han promovido en el ser humano la necesidad de seguir aprendiendo y actualizarse de manera continua, asimismo como la simplificación del estilo de enseñanza, estas herramientas han sido clave en la evolución en la educación. (Rivera, 2005).

La pedagogía establece variedades de técnicas que permiten ampliar la educación, el uso de esta ciencia aplicada a la educación ha servido para restaurar el sistema en donde se desenvuelve. Gracias a organizaciones como la UNESCO la cual se ha encargado de contribuir a la paz y a la seguridad en el mundo mediante la educación, la ciencia, la cultura y las comunicaciones, se han elaborado importantes estudios y estrategias para llevar a cabo estudios pedagógicos en todo mundo, teniendo énfasis en los países tercermundistas, donde las nuevas culturas de acción y reacción inmediata retraen la educación (Efland, 2003; Ramírez, 2008).

La pedagogía es una ciencia que permite nutrirse y aportar a distintas áreas de estudio, con la intención de buscar resultados y contribuir con la creación de nuevos programas de inclusión educativa, innovando en las distintas técnicas que ayuden a los docentes a impartir una mejor enseñanza a los estudiantes de todas las edades. Se utilizan materias de Ciencias sociales, historia, y la antropología como referencia para así poder comprender un poco más a la educación más como una filosofía en amplio crecimiento y no como un sistema (Corea, 2004; Giroux, 2005).

Está demostrado que la cultura y la pedagogía van completamente de la mano, por esto se recurrió a la combinación de la enseñanza con la lectura de figuras literarias como por ejemplo la leyenda del hombre caimán, pues este último representa la cultura que se debe cuidar en el departamento de Magdalena (Homs, 2004)

La importancia de la cultura radica en el hecho, que confiere identidad a una persona o grupo de personas que tienen relación histórica entre sí, permitiendo con ello conservar los elementos que fueron necesarios para su supervivencia en el planeta y mantenerlos como parte de sí, conocer nuestra cultura permite potencializar nuestras estrategias y adaptar elementos extranjeros sin perder la identidad, en un entorno de pluriculturalidad, permitiendo hacer aportes a la globalización quien otorga la movilidad de personas y conocimientos (Fanfani, 2018; Sanz, 2000).

Por su parte el lenguaje es por obvias razones la característica más marcada de la cultura, debido a que, el lenguaje ha sido el motivo de una larga historia de cambios y ha hecho parte de la tradición oral en nuestra cultura. Tambien es importante resaltar las diferentes formas de comunicarse con las demás personas, en las cuales de igual manera se incluye el acento (Tamayo, 2007).

Según Figueroa y Carrascal, (2016). Los procesos de lectoescritura son una necesidad actual, sin embargo, son las habilidades lingüísticas que presentan más falencias a nivel de educación básica y media en el país, lo cual se observa en los resultados de las pruebas de estado para medir el nivel educativo, donde el índice de desarrollo de pensamiento 
crítico de los estudiantes en las pruebas nacionales e internacionales, es muy bajo, sin embargo se ha observado que la educación a nivel rural, es muy poca, por lo cual los niveles de lectura y escritura son muy pobres.

El proceso de lectoescritura debe contemplar tres aspectos fundamentales; en primer lugar; la comprensión, que indica la forma en la que las personas interpretan y analizan el lenguaje oral y escrito, a su vez esta categoría implica habilidades como percepción del habla, conocimiento del léxico, la manera como se analiza la estructura gramatical de las oraciones y el discurso que en un texto refiere a la formulación y evaluación de conversaciones o textos extensos. En segundo lugar; la producción del habla, que indica el modo de producción de lenguaje, donde la producción busca conocer cómo se convierten los conceptos a su forma lingüística es más difícil. Y, en tercer lugar; el proceso de adquisición que indica cómo se aprende una lengua (Franco, Blanco y Cortes, 2013)

Según Vieiro y Gómez, (2004) la lectura es considerada como una actividad instrumental básica necesario para que el sujeto aprenda, interactúe y se comunique con las demás personas, mediatizando los contenidos de diferentes áreas del conocimiento a través del lenguaje oral y escrito, en ese sentido, la lectura no solo implica el entendimiento de los códigos gráficos, sino que se trata de la capacidad de elaboración y utilización de la lengua escrita, por lo tanto, ambos procesos van de la mano y no es posible estudiarlos de forma independiente porque se necesita de uno para adquirir el otro. Coincide con esta postura García y Arrieta (2016) cuando asumen la necesidad de fortalecer las competencias lectoras como base para el desarrollo cognitivo y metacognitivo; el perfil lector se convierte en un espacio de mediación para conferir significados a la realidad circundante.
Por otro lado, el entorno educativo, es un sistema organizado que permite un nivel de interacción entre estudiantes, docentes y cuerpo administrativo, sin embargo, desde los cursos iniciales de básica primaria, se puede observar un nivel de comunicación primitivo al momento de revisar las dificultades y falencias que se presentan dentro de las aulas de clase con los estudiantes sobre la adquisición de conocimientos que posibilita reconocer al otro a través de la escucha atenta junto a la percepción visual, encontrando la información necesaria para motivarlo a aprender el sistema estructurado de la lengua escrita y hablada, en ese sentido es necesario estimular el aprendizaje de la lectura y escritura en los primeros años, puesto que la curiosidad por desarrollarlos favorece el proceso de enseñanza, para esto utilizar la mediación de las tecnologías de la información puede ser una estrategia didáctica que permita la adquisición de habilidades en lectura y escritura. (Franco, 2010; Marín, Niebles, Sarmiento y Valvuena, 2017).

La forma en la cual los individuos se comunican entre sí, y comparten una serie de hechos y pensamientos particularmente heredado de generaciones pasadas, y en las cuales se puede citar por ejemplo al calendario azteca en el país de México, el simbolismo que representan las artes marciales en los países asiáticos, entre otros que tienen gran relevancia directa en la vida de los seres humanos; aunque en ocasiones llegan a ser actividades habituales, por lo tanto se convierten en rutina y son ignoradas . La escritura es el elemento más representativo (Díaz, 2001).

\section{Metodología}

\section{Diseño}

Se realizó un estudio de tipo cualitativo, utilizando el modelo de investigación acción, esta investigación busca conocer e 
interpretar la realidad de los participantes a través de sus propias experiencias, entregando una información subjetiva del fenómeno de estudio. Por su parte el modelo de investigación acción plantea una producción de conocimiento basado en la reflexión de los participantes, teniendo en cuenta la participación activa de maestros en el proceso de enseñanza, guiando al estudiante en su proceso de investigación. Generando nuevo saber y conocimiento sobre una realidad determinada en un proceso de aprendizaje colaborativo.

\section{Participantes}

Durante el estudio participaron ciento treinta (130) estudiantes con edades que oscilaban entre los siete (7) y once (11) años de la Institución Educativa Departamental Gabriel Escobar Ballestas, distribuidos en los cursos de tercero a quinto de primaria (Tabla 1).

TABLA 1

Distribución de los participantes

\begin{tabular}{cc}
\hline Grado & Número de Estudiantes \\
\hline Tercero & 35 \\
Cuarto & 45 \\
Quinto & 50 \\
TOTAL & $\mathbf{1 3 0}$ \\
\hline
\end{tabular}

Fuente: elaboración propia.

\section{Técnicas e instrumentos}

Dentro de las técnicas de recolección de datos se tomaron métodos propios de la investigación cualitativa, tales como, observación participante y herramientas como diario de campo donde se consignaron todas las experiencias pedagógicas, observadas en el proceso del fortalecimiento de la investigación como estrategia pedagógica a través de la lectura y escritura mediante figuras literarias.

\section{Procedimiento}

En base al diagnóstico realizado, sobre las dificultades presentadas por los estudiantes sobre lectura y escritura se diseñaron estrategias que fortalecieran al estudiante a usar la investigación como herramienta fundamental. A continuación, se presentan los diferentes momentos.

- Momento 1: conformación de los grupos de aprendizaje.

- Momento 2: se realizó una plenaria donde los estudiantes formularon preguntas generando grupos de discusión dando inicio al aprendizaje situado.

- Momento 3: el grupo planteo el problema, identificando las causas y efectos a nivel teórico mediante la representación de figuras literarias.

- Momento 4: se diseñaron los recorridos de indagación donde los estudiantes escogieron la diversidad metodológica y epistemológica para dirigir su proceso de investigación y aprendizaje, guiados por el docente.

- Momento 5: recolección de la información, contrastación y organización de la información a la luz de la teoría.

- Momento 6: síntesis de los resultados, reflexión de la práctica investigativa y apropiación del conocimiento a través de la socialización de sus experiencias en ferias institucional.

\section{Resultados}

Una vez definidos los grupos de investigación, de acuerdo a lo establecido desde la IEP, se definieron las preguntas del momento pedagógico 2 . Los estudiantes participaron activamente en la definición de las problemáticas más relevantes sobre las dificultades en la comprensión lectora. A partir de la plenaria se llegó a las siguientes conclusiones: 
- Uno de los estudiantes informó que los estudiantes no comprenden la lectura porque los temas que les toca indagar son muy académicos, le parece que la infromación es muy técnica, lo que imposibilita el disfrute y apropiación de los conceptos clave.

- En términos generales, los niños consideran que es necesario practicar en puntuación, pronunciación y entonación de la lectura desde sus aspectos más técnicos, con el fin de poder entender la idea de los autores que revisamos y sea posible el análisis de cada frase contenida en el mismo.

- Se identifica la necesidad de realizar lecturas menos profundas: Uno de los niños dijo: "Es que si leyéramos un cuento o un artículomás divertido, nos gustaría más".

- Otro problema que se identificó fue que los profesores no verifican que los niños hayan entendido las lecturas revisadas. Sugiernen la posibilidad de hacer controles de lectura con más frecuencia y trabajos más orientados al análisis de material relacionado con aspectos sociales y culturales de las comundiades donde se desenvuelven los estudiantes.

- En el momento 3 del proceso pedagógico de la IEP, los estudiantes identificaron causas y efectos de los problemas de comprensión lectora en la institución. Entre los más significativos se pueden considerar los siguientes:

- El poco acompañamiento de los docentes en lo que respecta a la verificación del desarrollo de las competencias, la identificación de fortalezas, debilidades, amenazas y oportunidades.

- El bajo rendimiento académico de algunos estudiantes puede deberse a la poca comprensión de lectura, que imposibilita el aprendizaje efectivo de los contenidos curiculares.

- El grupo de estudiantes ideó como estrategia de resolución de las dificultades en comprensión lectora, el uso de figuras literarias (que los estudiantes pudieras redactar el mismo material a leer e interpretar en las aulasde clase, a partir de sus experiencias en el municipio donde habitan y aspectos culturales y sociales que dinamizan los proesos de interacció entre todos.
Los momentos 4 y 5 incluyeron el diseño de acciones encaminadas a promover la lectura y escritura a través de figuras literarias, pues los estudiantes lograron redactar sus propios cuentos inspirándose de la lectura que habían realizado previamente de las leyendas propias de su región, para ello, los estudiantes se organizaron en grupos de aprendizaje colaborativo, donde mediante el diálogo de saberes entre ellos y actores sociales de la comunidad, lograron conocer las historias sobre los mitos y leyendas del municipio, a través de la observación después de realizar la recolección de información con la comunidad se realizó el recorrido de las trayectorias de indagación, donde los estudiantes contrastaron la información obtenida en los diarios de campo con la teoría, realizando una revisión literaria.

En total se diseñaron 5 libros (uno para cada grado escolar de primaria), supervisado por un director de grupo del grado correspondiente. El material fue entregado a los estudiantes con el propósito de ser revisado en un período de un mes y luego hacer un club de lectura.

El momento 6 del proyecto, permitió que los estudiantes obtuvieran sus propias conclusiones, partiendo del análisis de la experiencia. Los resultados obtenidos en el proceso se presentaron en los clubes de lectura de los siguientes 5 meses, en los que fue posible evidenciar un compromiso y vinculación por parte de los estudiantes con los procesos de lectura y escritura, así como mejores calificaciones y más compromiso de comprensión del material proporcionado.

\section{Discusión}

El conocer e identificar las leyendas o figuras literarias de la región del Magdalena, permitió despertar en los estudiantes el interés por la investigación, la lectura, la redacción de cuentos y el amor por la cultura. Los estudiantes como producto, pudieron realizaron un cuento basándose en lo leído de las leyendas, tomando como referencia la lectura del hombre caimán, para efectuar así una investigación sobre cómo realizar tal obra. La implementación de un proyecto pedagógico para promover el interés por las clases por parte de los estudiantes, es una muy buena 
forma de captar su atención, pues con este proyecto se logró hacerlo e incluso enseñarlos a construir su propia historia y plasmarla en un cuento.

En ese sentido, los resultados anteriormente descritos convergen con los planteamientos de autores como Ortega, Peñuela y López (2009). Los cuales comentan, que, al pensar en la investigación desde las corrientes educativas críticas, permite una lucha teórica practica en relación a sus supuestos sobre el conocimiento, ciencia, epistemología, cultura, humano y grupos sociales, enmarcando acciones las cuales se han señalado como objetiva, ayudando así a generar formas de autoridad en la sociedad las cuales sirven para el control y generación de desigualdades, y en la actualidad, nuevas de dominio.

\section{Referencias}

Acevedo, C. (2017). Las tiendas de barrio desde la economía institucional. International Journal of Management Sciences and Operations Research, 2(1), 30-37. Recuperado a partir de http://ijmsoridi.com/ index.php/ijmsor/article/view/85

Benito, A. y Escolano, A. (2002). La educación en la España contemporánea: politicas educativas, escolarización y culturas pedagógicas. Madrid: Biblioteca Nueva.

Camargo, C. (2015). La investigación como estrategia pedagógica en la Guajira, desde una perspectiva de la inclusividad y diversidad como nuevo paradigma para el desarrollo de habilidades, destrezas y competencias. Educación y ciudad, (29), 149-162.

Carvajal, P. (2013). El reconocimiento de derechos a la comunidad LGBTI. Juridicas CUC, 9(1),123-141.

Corea, C. y Lewkowicz, I. (2004). Pedagogía del aburrido. Escuelas destituidas, familias. Buenos Aires: Paidós.

Díaz, R. (2001). Trabajo docente y diferencia cultural: lecturas antropológicas para una identidad desafiada. Quito: Abya Yala.

Efland, A., Freedman, K. y Stuhr, P. (2003). La educación en el arte posmoderno. Barcelona: Paidós.
Fanfani, E. (2018). Culturas juveniles y cultura escolar. Revista Colombiana de Educación, (40-41). https://doi. org/10.17227/01203916.7772

Figueroa, H. y Carrascal, A. (2016). Potencialización del pensamiento crítico a través de la Hipotemetacomprensión Textual (HMT). Cultura Educación y Sociedad, 7(2), 54-71.

Franco, M. (2010). Estrategias de enseñanza y la comprensión lectora. Revista Cultura, Educación, Sociedad-CES, 1(1). 53-64.

Franco, M., Blanco, P. y Cortés, O. (2013). Papel de las habilidades metalingüísticas en los procesos de lectura y escritura en la educación superior. Escenarios, 11(2), 82-86.

Freire, P. (2005). Pedagogía del oprimido. México, D.F.: Siglo XXI.

Gadotti, M. (2002). Historia de las ideas pedagógicas. México, D.F.: Siglo XXI.

García, H. y Arrieta, A. (2016). Potencialización del Pensamiento Crítico a través de la Hipotemetacomprensión Textual (Hmt). Cultura Educación y Sociedad, 7(2), 54-71. Recuperado de https://revistascientificas.cuc.edu.co/ culturaeducacionysociedad/article/ view/1102

Giroux, H. A. (2005). Estudios culturales, pedagogía crítica y democracia radical Madrid: Popular.

Giroux, H. y McLaren, P. (2003). Por uma pedagogia crítica. Territórios contestados: o currículo e os novos mapas politicos e culturais, 5(1), 144-158.

Hernández, D. y Escobar, A. (2017). Modelo de contabilidad social como herramienta de gestión para la responsabilidad social empresarial. IJMSOR, 2(1), 44-56. Recuperado de http://ijmsoridi.com/index.php/ijmsor/ article/view/86

Kincheloe, J. (2008). La pedagogía crítica en el siglo XXI: Evolucionar para sobrevivir. En, P. McLaren (Coord.), Pedagogía crítica. De qué hablamos, dónde estamos, (25-69). Barcelona: Graó. 
Marín, F., Niebles, M., Sarmiento, M. y Valvuena, S. (2017). Mediación de las tecnologías de la información en la comprensión lectora para la resolución de problemas aritméticos de enunciado verbal. Revista Espacios, 38(20). 20.

Mejía, M. y Manjarrés, M. (2010) La Investigación como Estrategia Pedagógica. Bogotá, D.C.: Ondas-Colciencias.

Moreno, G. (2015). Una Aproximación al concepto de Observatorio Social. Cultura Educación y Sociedad, 6(1). 93.108. Recuperado de https://revistascientificas. cuc.edu.co/culturaeducacionysociedad/ article/view/758

Muñoz, C. (2004). Pedagogia da vida cotidiana e participação cidadã. São Paulo: Cortez.

Nieves, J. y Salazar, F. (2016). e-Supply chain management para una empresa de tecnología. IJMSOR, 1(1), 31-34. Recuperado de http://ijmsoridi.com/index. php/ijmsor/article/view/74

Ortega, P., Peñuela, D. y López, D. (2009). Sujetos y prácticas de la pedagogía crítica. Bogotá, D.C.: El Búho.

Pastor, M. (2004). Pedagogía museística: nuevas perspectivas y tendencias actuales. Barcelona: Ariel.

Pino, M. y Urrego, Y. (2013). La importancia de las funciones ejecutivas para el desarrollo de las competencias ciudadanas en el contexto educativo. Cultura Educación y Sociedad, 4(1). 9.20. Recuperado de https://revistascientificas.cuc. edu.co/culturaeducacionysociedad/article/view/969

Pinto, M. y Misas, M. (2014). La educación inicial y la educación preescolar: perspectivas de desarrollo en Colombia y su importancia en la configuración del mundo de los niños. Cultura Educación y Sociedad, 5(2). 119-140. Recuperado de https://revistascientificas.cuc.edu.co/culturaeducacionysociedad/article/ view/889

Ramírez, R. (2008). La pedagogía crítica: Una manera ética de generar procesos educativos. Folios, (28), 108-119.
Rivera, J. (2005). La contextualización de la cultura en los currículos de las carreras pedagógicas. [Doctoral dissertation]. Instituto Superior Pedagogico "Frank País García, Santiago de Cuba.

Sacristán, J. (2001). Educar y convivir en la cultura global: las exigencias de la ciudadanía. Madrid: Motora.

Santacoloma-Suárez, A. (2012). Los semilleros de investigación como estrategia de formación integral para ciudadanos del tercer milenio. Cultura Educación y Sociedad 3 (1): 13-21.

Sanz, T. y Rodríguez, M. (2000). El enfoque histórico cultural: su contribución a una concepción pedagógica contemporánea. En, Cepes (Colectivo) Tendencias pedagógicas en la realidad educativa actual. Tarija: Editorial Universidad Juan Misael Saracho.

Segura, E. (2016). Información, estabilidad y complejidad de aprendizaje en memorias asociativas. IJMSOR, 1(1), 49-53. Recuperado a partir de http://ijmsoridi. com/index.php/ijmsor/article/view/77

Sepúlveda, A., Martínez, R., Medina, S. y Salazar, F. (2016). Propuesta de diseño de una red supply chain para la agrocadena de cacao, municipio de Viotá, Cundinamarca. IJMSOR, 1(1), 35-42. Recuperado de http://ijmsoridi.com/index.php/ijmsor/article/view/75

Tamayo, L. (2007). Tendencias de la pedagogía en Colombia. Revista Latinoamericana de Estudios Educativos (Colombia), 3(1). 65-76.

Uribe, J. (2002). Historia de la pedagogía como historia de la cultura. Bogotá, D.C.: CESO.

Vieiro, P. y Gómez, I. (2004). Psicología de la lectura: procesos, teorías y aplicaciones instruccionales. Madrid: Pearson Educación, S. A. 